\title{
Protéger et contrôler la présence et les activités des étrangers dans les villes portuaires du nord de la Couronne de Castille au Moyen Âge
}

Jesús Ángel Solórzano Telechea et Beatriz Arízaga Bolumburu

\section{(2) OpenEdition \\ 1 Journals}

\section{Édition électronique}

URL : http://journals.openedition.org/abpo/1021

DOI : $10.4000 /$ abpo. 1021

ISBN : 978-2-7535-1517-8

ISSN : 2108-6443

\section{Éditeur}

Presses universitaires de Rennes

\section{Édition imprimée}

Date de publication : 20 avril 2010

Pagination : 209-222

ISBN : 978-2-7535-1146-0

ISSN : 0399-0826

\section{Référence électronique}

Jesús Ángel Solórzano Telechea et Beatriz Arízaga Bolumburu, «Protéger et contrôler la présence et les activités des étrangers dans les villes portuaires du nord de la Couronne de Castille au Moyen Âge ", Annales de Bretagne et des Pays de l'Ouest [En ligne], 117-1 | 2010, mis en ligne le 20 avril 2012, consulté le 02 mai 2019. URL : http://journals.openedition.org/abpo/1021 ; DOI : 10.4000/abpo.1021

Ce document a été généré automatiquement le 2 mai 2019.

(c) Presses universitaires de Rennes 


\title{
Protéger et contrôler la présence et les activités des étrangers dans les villes portuaires du nord de la Couronne de Castille au Moyen Âge
}

\author{
Jesús Ángel Solórzano Telechea et Beatriz Arízaga Bolumburu
}

1 Les connaissances dont nous disposons sur les étrangers dans la Castille médiévale sont peu nombreuses, en particulier pour les villes portuaires du nord de la péninsule. Depuis les travaux pionniers de Jules Finot à la fin du xixe siècle l'étude de la présence des étrangers dans le royaume de la Castille au Moyen Âge s'est heurtée à la pénurie documentaire du fait qu'ils n'avaient pas l'habitude de se grouper en consulats. On trouve surtout leur trace dans la documentation judiciaire, ce qui empêche toute étude systématique. Le rôle des Génois en Andalousie, notamment à Séville et à Cordoue, est assez bien connu mais nous ne savons presque rien sur les Flamands, les Français, les Anglais et les Portugais ${ }^{1}$. Cela ne signifie pas pour autant que leur présence ait été un phénomène marginal comme l'ont montré plusieurs historiens².

\section{La présence étrangère dans les ports du nord de la Castille}

2 Les étrangers sont présents dans les villes portuaires du nord de la Castille dès le début de XIII ${ }^{\mathrm{e}}$ siècle, dès lors que les marchands de ces villes commencèrent à tisser des liens humains et commerciaux avec les ports de la façade atlantique européenne, en particulier ceux du golfe de Gascogne. On trouve des Anglais, des Français et des Gascons à SaintSébastien, Santander, La Corogne et Avilés au XIII ${ }^{\mathrm{e}}$ siècle, tels les Guiralt, Arnaot, Brones, Amat, Burges, Caspín, Mathe, Bernalt, Godofré, Guillen de Flaias, Bernardin, Rogel, Prinalt, Rinalt à Santander, ou encore Guillem Per de Mans, Per de Nordmech, Dominique 
de Mans ou d'Odicheu à Saint-Sébastien. Il s'agissait des marchands étrangers appelés francos, attirés par les privilèges économiques concédés par les rois de Castille ${ }^{3}$.

Passé les premières années qui suivirent la fondation de ces villes, les contacts entre les villes portuaires cantabriques et les ports atlantiques et méditerranéens n'ont pas attiré d'autres étrangers. Il faut attendre la fin $\mathrm{du} \mathrm{Xv}^{\mathrm{e}}$ siècle pour voir des marchands anglais et flamands ou leurs facteurs étrangers s'établir dans les villes portuaires grâce à l'intensification des relations entre les ports anglais et cantabriques. Après la perte de la Gascogne, l'Angleterre cherchait à s'approvisionner en Castille en sel, vin, fer et produits de luxe. Parfois il s'agit de facteurs étrangers établis temporairement. William Botyller opéra à Bilbao entre 1480 et 1490 en qualité de facteur de William Hadden. Après la mort de ce dernier, il voulut rester dans la ville mais il fut accusé d'avoir utilisé ses amis de Bilbao pour tromper les héritiers de Hadden. À Laredo et à Bilbao, Cornelis Deque, marchand néerlandais habitant Valladolid, disposait de l'appui d'un facteur issu de la même nation, Jean de Ypre, nanti depuis 1475 d'une lettre de sauvegarde royale pour commercer et qui importait des produits flamands à Valladolid 4 .

4 L'intégration des étrangers était profonde quand elle se produisait. Ce fut le cas de Thomas Batcok, facteur du marchand londonien Thomas Howell, qui avait une famille à Rentería et dont les enfants ignoraient la langue anglaise ${ }^{5}$, ou celui du marchand anglais Huatre Tunson (Thomson), voisin de Deva ${ }^{6}$, garant du reste des voisins de cette ville et qui fut emprisonné pour défaut de comparution lors d'un procès en $1518^{7}$. Il y avait aussi des étrangers qui, intégrés localement, travaillaient pour leur pays d'origine à l'exemple de Tomás Traves, marchand anglais, habitant Bilbao au début du xvI siècle. Il gardait les prisonniers français par ordre de l'ambassadeur d'Angleterre et intenta un procès à deux voisins d'Eibar qui avaient aidé les prisonniers à s'enfuir ${ }^{8}$. La documentation judiciaire livre les noms de nombreux marchands étrangers : Guillem Brum, anglais, voisin de SaintSébastien; Rubete Uxun, Juan Junarque, Guillemo Lebrón, Guillén Escaller, Cristóbal Rengente, Mateo Lambert, Guillem Chinchi, Tomás Oloed, Ullen Church et Juan Treque, marchands anglais, habitant Bilbao entre la fin du $\mathrm{Xv}^{\mathrm{e}}$ et le début du $\mathrm{XvI}^{\mathrm{e}}$ siècle ${ }^{9}$. Ces marchands établis dans les ports du nord étaient en relation d'affaires avec des marchands de même nationalité installés dans d'autres villes de Castille, ce qui révèle l'existence de réseaux commerciaux s'étendant vers l'intérieur du pays et conforte l'idée de marchands intégrés dans des groupes et des réseaux de commerce ${ }^{10}$. Niculas Montaot, voisin de Fontarabie, et Guillen Holibru, marchand de Séville, agissant au nom de marchands anglais, dénoncèrent par exemple un vol de marchandises perpétré par des Français à Bayona en Galice ${ }^{11}$. Tous les étrangers n'étaient pas marchands. En 1516, la ville de Bilbao engagea Philippe Picard, originaire de Picardie, pour amener l'eau de la rivière jusqu'à la ville afin de nettoyer les rues et les cours et de renvoyer ensuite l'eau sale à la rivière. Le contrat nous apprend que Bilbao était alors une ville superpeuplée sans sol urbain libre, avec beaucoup d'étrangers qui habitaient des maisons aussi hautes et étroites que celles des voisins. Les gens jetaient les immondices dans les rues, ce qui nuisait à l'hygiène et à l'image de Bilbao. Philippe Picard accepta le marché mais demanda au conseil municipal une lettre de sauvegarde pour lui et ses biens en cas de guerre ${ }^{12}$.

5 À côté des étrangers originaires de France et d'Europe du Nord, d'autres venaient du Sud. C'était le cas d'Andres Abelat, Florentin, habitant de Bilbao, associé à Pierre de Vanengue, tapissier de Philippe Le Beau ${ }^{13}$. D'autres étrangers sont repérables par leur nom qui indique une origine géographique : Breton, Bourgogne, Bayonne, Flamand, Allemand, Anglais, Franc, Corse, Génois... On rencontre aussi le cas de Français qui, en 1523, possédaient des 
terres dans la juridiction des Quatre Villes de la Côte de la Mer. Ferdinand de Setién, voisin de Santander, demanda à Charles v la mise sous séquestre de leurs biens et pria qu'on les lui attribue en considération de ses mérites militaires car il avait fidèlement servi le roi dans ses guerres, en particulier à Fontarabie où il avait perdu la main droite. Le monarque ordonna d'enquêter sur la situation économique et familiale des Français. Il apparut alors qu'ils étaient pleinement installés dans les villes, raison pour laquelle Ferdinand de Setién obtint seulement 5000 maravédis de compensation ${ }^{14}$.

\section{Protéger et contrôler les étrangers}

6 Le concept d'étranger recouvre des situations diverses ${ }^{15}$. À côté des étrangers de passage comme les pèlerins, les marchands et les vagabonds, qui étaient les plus nombreux, figurait un petit nombre d'étrangers établis dans les villes castillanes pour exercer leur métier, sans être naturalisés castillans ou bien ayant leur résidence dans une autre ville. En Castille, sous l'influence du droit romain, la législation leur était très favorable. Elle privilégiait la notion d'origine face au droit coutumier qui s'attachait au domicile.

7 La présence et les activités des étrangers en Castille étaient régulées par les coutumes et le droit royal qui déterminaient leur statut dans les villes. La législation castillane facilitait l'accueil de certains étrangers, notamment les marchands et tous ceux qui semblaient utiles à la société. En effet, la plupart des étrangers dans les villes portuaires étaient des marchands originaires des royaumes riverains de la côte atlantique ${ }^{16}$. Leur situation juridique en Castille changea avec Alphonse X(1252-1284). Ce roi assouplit le protectionnisme qui avait prévalu pendant le règne de son père Ferdinand III (1217-1252). Ce changement visait à augmenter les revenus royaux et à faire droit à la revendication de la noblesse désireuse d'exporter certains produits comme la laine ${ }^{17}$. Las Partidas stipulent que les rois étaient obligés « d'aimer et d'honorer » les marchands étrangers qui approvisionnaient le royaume en produits indispensables ${ }^{18}$. Ce code reprend quarante normes spécifiques qui établissaient un cadre juridique propice au développement de l'activité commerciale des marchands étrangers en Castille. Alphonse $\mathrm{X}$ a traité une ample gamme de sujets relatifs à la sécurité des activités mercantiles (commerce et guerre sur mer, fret, naufrages, produits prohibés, contrats d'achat, prêts, etc.). De la même façon, il a légiféré sur les marchandises, les personnes et les familles des marchands étrangers. En 1281, il les exempte du paiement de droits dans les ports pour les biens destinés à leur usage et à celui de leur famille ou pour les cadeaux aux «bons hommes» des conseils municipaux, à la condition qu'ils ne les vendent pas ensuite ${ }^{19}$. La volonté du roi de favoriser, protéger et défendre les activités des marchands étrangers était liée aux revenus de plus en plus considérables qu'elles fournissaient à la Couronne et aux villes portuaires. À cet effet Alphonse x instaura la Dîme de la Mer de la Castille qui grevait l'entrée et la sortie de toutes les marchandises par les ports du golfe Gascogne d'un droit ad valorem de $10 \%$. De ce point de vue, la protection accordée par Alphonse X assimilait les étrangers à d'autres minorités comme les Juifs et les Mudéjars ${ }^{20}$.

8 Les autres lois et coutumes qui s'imposaient aux étrangers dans les villes du nord péninsulaire étaient les Rôles d'oléron, le Fuero Real de 1254 et l'ordonnance d'Alcala de Henares de 1348 régissant les affaires maritimes. Les marchands étrangers connaissaient la législation castillane et savaient en faire usage. En 1480, Robert Ochoa, bourgeois de Bristol, demanda aux Rois Catholiques de lui délivrer des lettres de sauvegarde et de 
protection au titre de la protection qu'Alphonse $\mathrm{X}$ et Alphonse XIavaient établie respectivement dans Las Partidas et l'ordonnance d'Alcala de Henares ${ }^{21}$.

Les rois eurent aussi à légiférer avec les Cortes pour protéger les étrangers. Lors des Cortes de Toro de 1371, Henri II déclara les étrangers qui venaient à vivre en Castille libres du paiement de tout droit pendant les dix premières années de leur séjour ${ }^{22}$. Les mesures royales répondaient parfois aux requêtes des procureurs des villes qui demandaient la limitation des activités des étrangers. Les Cortes de Madrid de 1419 établirent que « les Gascons, les Navarrais, les Aragonais ou autres étrangers étaient obligés de déclarer les marchandises importées » aux agents de la douane et il leur était défendu de faire sortir du royaume de l'or, de l'argent, des chevaux ou des mules ${ }^{23}$.

10 Les Rois Catholiques ont poursuivi la politique bullioniste de leurs prédécesseurs tout en veillant à préserver les transactions réalisées par les étrangers. Les sorties d'or et d'argent $d u$ royaume par les ports cantabriques étaient une des principales préoccupations. En 1488, un ordre royal envoyé aux villes portuaires de Guipúzcoa imposait d'inventorier les marchandises étrangères importées par les étrangers vivant dans le royaume et obligeait ces derniers à convertir leurs profits en produits locaux afin qu'ils ne fassent pas sortir de métal précieux du royaume. Cet ordre fut réitéré dans les ports de Biscaye et dans les Quatre Villes de la Côte de la Mer en 1491: "Quelques étrangers de nos royaumes venaient dans les ports des villes avec beaucoup de tissus et marchandises et autres choses, lesquelles ils vendaient toutes à une monnaie d'or et d'argent et ils les tiraient et portaient outre mes royaumes. Et ainsi ils tiraient des pastels et des vins de Bordeaux et de Bayonne et d'autres parts ${ }^{24}$.» Cependant, des assouplissements furent accordés afin de favoriser l'approvisionnement des villes portuaires toujours déficitaires en céréales. En 1476, les Rois Catholiques permirent aux habitants de Bilbao de payer en argent les céréales importées mais en 1484 ils les punirent pour avoir acquitté en numéraire des importations de grains ${ }^{25}$. La contrebande permettait de tourner les interdictions. À Laredo, au début du $\mathrm{xVI}^{\mathrm{e}}$ siècle, un conflit opposa des Bretons à la justice locale. Alors qu'ils se disposaient à repartir pour la Bretagne après avoir approvisionné la ville en céréales, ils furent attaqués et leurs biens saisis par les autorités. Le lieutenant du corregidor les interrogea par l'intermédiaire d'interprètes pour connaître la quantité d'argent qu'ils emportaient et confisca les marchandises et le bateau. Les Bretons alléguèrent pour leur défense qu'on ne pouvait pas séquestrer le navire et l'argent sans une sentence judiciaire. La justice de Laredo ayant retardé le procés, les Bretons en appelèrent à la justice royale à Valladolid ${ }^{26}$.

11 Les dispositions prises par les Rois Catholiques pour contrôler l'activité commerciale se renforcèrent dans les années 1490. Ils défendirent aux marchands étrangers d'acheminer vers un pays ennemi le fer et l'acier castillans. En 1497, en raison de l'importance de ces produits pour l'économie de la province, les Rois Catholiques permirent aux Guipuzcoans de vendre du fer et de l'acier aux marchands anglais, à condition qu'il ne soit pas ensuite revendus aux Français ${ }^{27}$. En 1499, ils imposèrent aux marchands locaux et étrangers de charger leurs marchandises sur des navires castillans avant ceux des forains. Mais l'année suivante, le Guipúzcoa et la Biscaye furent dispensés de cette obligation ${ }^{28}$. En 1501, il fut interdit de vendre des navires aux étrangers, même une fois naturalisés, sans autorisation préalable $^{29}$. Il s'ensuivit des litiges. En 1507, Esteban de Amasa accusa Marticot de la Salle, tous deux étant voisins de Fontarabie, d'avoir vendu la moitié d'une nef à Gilles Mems, Anglais, en violation de la Pragmatique de $1501^{30}$. 
12 Les mesures répressives prises par les Rois Catholiques eurent pour effet de dissuader les marchands étrangers de venir dans les ports du nord de la Castille. En 1517, la municipalité de Portugalete se plaignait que les étrangers refusaient de se rendre en Castille. Elle dénonçait l'application stricte à Bilbao de la loi de 1488 obligeant les étrangers à déclarer les marchandises importées et à fournir des cautions pour garantir qu'ils exporteraient leurs gains en produits castillans non prohibés. La reine Jeanne donna raison à Portugalete mais elle fut blâmée par le conseil municipal de Bilbao ${ }^{31}$. La politique protectionniste castillane nuisait à la circulation des personnes et des marchandises. En 1476, Juan de Mole, bourgeois de Saint-Pol-de-Léon, sollicita la protection des Rois Catholiques. À la suite de Pierre Bilbao aucun autre voisin de Portugalete n'avait voulu se porter garant pour lui, compte tenu de sa condition d'étranger ${ }^{32}$.

13 Malgré les dispositions restrictives prises pour contrôler les activités économiques des étrangers en Castille, les Rois Catholiques les ont protégés par des traités internationaux et des lettres d'assurance en particulier en période de guerre. En 1476, les conseils municipaux de la Principauté des Asturies remontrèrent qu'ils avaient besoin de sel pour saler la viande et le poisson, produits dont le commerce soutenait l'économie de ces villes. À cause de la guerre que le Portugal et la France faisaient à la Castille, les marchands qui approvisionnaient les greniers à sel n'osaient plus venir. Les Rois Catholiques accordèrent une lettre d'assurance à « n'importe quel marchand étranger des dits royaumes de France et du Portugal, pour qu'ils puissent venir et être sûrs dans la dite ville d'Oviedo et dans les autres villes et les lieux et nos mers de la Principauté avec ses navires, avec tout le sel et d'autres choses avec la condition de ne pas pouvoir tirer de nos royaumes les dits chevaux et les armes et les autres choses qui sont défendues ${ }^{33}$ ». En 1478, Ferdinand ordonna aux villes portuaires du nord de la péninsule de respecter les personnes et les biens des Anglais $^{34}$. En 1488, les Rois Catholiques demandèrent au corregidor de Guipúzcoa d'enquêter sur l'habitude qu'avaient "Bayonne, la Terre de Labourd et de Capbreton " d'une part, Fontarabie et Saint-Sébastien d'autre part, de ne pas exécuter dans leurs territoires respectifs les lettres de marque et de représailles contre les étrangers ${ }^{35}$. En 1509, Bilbao se plaignait du manque de denrées de première nécessité car les étrangers n'osaient pas venir à cause des lettres de course de telle façon que la ville pouvait se dépeupler. La reine Jeanne décréta la protection des marchands étrangers qui approvisionnaient Bilbao et annula les lettres de marque en vigueur ${ }^{36}$.

La Castille avait passé des accords de protection mutuelle avec les principaux royaumes méditerranéens et atlantiques. Quand des particuliers ou les autorités locales ne respectaient pas ces conventions, les affectés réclamaient justice au roi. En 1491, Micer Ulises, agissant au nom de plusieurs marchands vénitiens, accusa Ferdinand de Escalante et Jean d'Augure, voisins de Santander, d'avoir assailli et pris des marchandises estimées à 30000 ducas d'or bien qu'ils soient venus en Castille "sous l'assurance de paix et d'amitié qu'il y avait entre nous et nos royaumes et la communauté des Vénitiens ${ }^{37}$ ». En 1480, la justice royale fut saisie par des marchands anglais habitant Bilbao, représentés par André Ingles. Ils protestaient contre l'impôt exigé d'eux par la ville, en violation des accords entre la Castille et l'Angleterre selon lesquels les Anglais ne devaient pas payer plus de taxes que les Castillans. Le procureur du conseil municipal, Juan Sánchez de Arbolancha, fit valoir que le nouveau tribut était destiné à la réparation des ponts, fontaines, murs et quais de la ville, et que les Anglais établis à Bilbao étaient tenus de 
contribuer comme le reste des voisins puisqu'ils étaient « habitants et résidants dans la ville ${ }^{38} »$.

Les marchands étrangers constituaient un collectif privilégié par les lettres d'assurance du roi. Ils traitaient directement avec l'administration roayle pour l'obtention de celles-ci ou avec l'aide de voisins. En 1489, les Rois Catholiques accordèrent une lettre d'assurance à Robert Petet, marchand anglais, et à ses facteurs Adán Haemelio, Jean Braner et Jaymes Jaspes, parce qu'ils craignaient d'être maltraités ${ }^{39}$. Les lettres d'assurance pouvaient être achetées, comme celles acquises par Pedro de Llanes, voisin de Saint-Sébastien, pour le compte de trois marchands de La Rochelle ${ }^{40}$. Les personnes munies d'une lettre d'assurance saisissaient les tribunaux en cas de non respect de la protection accordée. En 1483, au terme d'un procès, les Rois Catholiques ordonnèrent à Jean de Zabalza et à ses amis, voisins d'Ondárroa et de Deva, de rendre à Jean Macosin, marchand écossais, les marchandises et le vaisseau qu'ils lui avaient pris ${ }^{41}$.

En dernier recours, les marchands étrangers comptaient sur l'appui des diplomates. En 1477, les ambassadeurs du roi de l'Angleterre se plaignirent de vols et réclamèrent justice. Ferdinand donna pouvoir au bachelier Jofre de Sausola pour résoudre l'affaire avec le corrégidor de la Biscaye et l'assistant de Guipúzcoa ${ }^{42}$. Comme le reste des étrangers, les ambassadeurs pouvaient eux aussi être victimes de violences. En 1492, des ambassadeurs anglais furent injuriés, agressés et enfermés dans l'auberge du port de Pasajes. Le prévôt de Saint-Sébastien qui avait pris le parti des agresseurs, en dépit de l'intervention du maire de la ville pour protéger les Anglais, fut destitué ${ }^{43}$.

\section{Les gouvernements locaux face à la présence étrangère}

Le séjour et l'installation des étrangers dans les villes étaient soumis à l'autorisation du conseil municipal, chargé de veiller à la sécurité de la population, de garantir la réparation des dommages possibles, de protéger la stabilité des transactions mercantiles et de faire appliquer la législation générale du royaume.

condition d'étranger constituait un motif substantiel de différenciation entre les étrangers et les voisins. Les lois royales et la réglementation urbaine plaçaient les étrangers dans une position désavantageuse sur le plan économique, social et politique par rapport aux voisins. Les étrangers ne pouvaient pas participer à la défense et au gouvernement de la ville. L'obtention d'une charge publique était subordonnée au fait d'être naturel du royaume et voisin résidant dans la ville. Les représentants urbains aux Cortes protestaient contre les libéralités royales consenties aux étrangers. En 1419, lors des Cortes de Madrid, les villes demandèrent que les postes municipaux soient réservés à des personnes natives du royaume ou qui y habitaient depuis dix ans au moins ${ }^{44}$. Ils réitérèrent leur demande aux Cortes de Palenzuela (1425) et de Burgos (1430) ${ }^{45}$. Les étrangers non naturalisés et mariés n'étaient pas intégrés dans les sociétés urbaines et, par conséquent, n'appartenaient à aucun lignage. Cela posait un problème à l'oligarchie urbaine en place et dont les lignages se partageaient entre eux les charges municipales ${ }^{46}$. Les ordonnances de Laredo établissaient trois conditions d'éligibilité pour devenir membre du conseil municipal : être marié, avoir une maison et résider dans la ville ${ }^{47}$.

Les ordonnances urbaines réglaient les activités économiques des étrangers dans les villes et les taxaient au profit de la municipalité. Les étrangers ne pouvaient pas disposer 
librement des ressources naturelles de la ville et bénéficier de sa juridiction contrairement aux voisins. Les ordonnances de Castro Urdiales établissaient que les étrangers qui voulaient couper du bois ou prendre des pierres pour lester les navires devaient payer pour cela alors que les voisins étaient exempts ${ }^{48}$. Les marchands étrangers devaient respecter des règles pour pouvoir accéder au marché urbain. Les ordonnances de Bilbao de 1477 obligeaient " Anglais, Bretons, Flamands, Français et autres étrangers » à déclarer leurs marchandises devant le député des marchands à l'entrée et à la sortie de la ville. Les aubergistes avaient l'obligation de leur demander ladite déclaration avant de les accueillir dans leur établissement. Les ordonnances de Santoña de 1445 permettaient l'entrée des navires étrangers dans le port mais leur interdissaient de charger et décharger les marchandies sans la licence de la municipalité ${ }^{49}$.

Les conseils municipaux de Castro Urdiales et Deva essayèrent d'éviter les fraudes lors des ventes de marchandises par les étrangers. Une "ordonnance ancienne" de Castro Urdiales établissait que l'achat de marchandises étrangères devait se faire en public et avec une licence municipale dans le cas d'une vente au détail ${ }^{50}$. En 1434, les ordonnances de Deva obligeaient à débaler les marchandises et à les porter au marché public où le conseil fixait le juste prix ${ }^{51}$. Les mesures prises contre les revendeurs avaient pour but d'éviter la spéculation et la hausse des prix. Elles entendaient donner la préférence aux voisins pour l'achat des céréales (froment, orge et seigle). Certaines ordonnances limitaient le nombre de jours pendant lesquels les étrangers pouvaient acheter des marchandises. À Bilbao, d'après les ordonnances de 1477, ils ne pouvaient acheter que les mercredis ${ }^{52}$. À Castro Urdiales, les étrangers payaient des droits d'entrée doubles de ceux des voisins pour le vin et le cidre ${ }^{53}$. Les conseils municipaux voulaient que les marchands étrangers contribuent aux frais urbains au même titre que les voisins. En 1519, le conseil municipal du Bilbao se plaignait des étrangers qui exerçaient leur métier sans permission municipale et quittaient la ville sans rien payer. Il fut décidé que les étrangers désireux d'exercer leur métier à Bilbao devaient obtenir la permission municipale et s'engager à résider dans la ville pendant au moins sept ans avec les mêmes obligations que le reste des voisins. Pour devenir voisin, il leur en coûtait 500 maravédis $^{54}$.

21 Les aubergistes qui hébergeaient les marchands étrangers servaient d'intermédiaires et jouaient un rôle important dans les transactions commerciales ${ }^{55}$. Ils offraient aux marchands le gîte et le couvert et entreprosaient leurs marchandises. Ils pouvaient à l'occasion aider à trouver des garants ou des clients, faciliter les paiement des taxes. Ce fut le cas de Pero Yáñez de Novia qui hébergea Jean Ingles en $1480^{56}$. Les aubergistes redistribuaient en gros ou au détail une partie des marchandises des marchands étrangers. Dès la fondation des villes, l'activité de ces professionnels qui travaillaient au plus près des marchands fut réglementée. La Coutume du Saint-Sébastien (vers 1180) réglait le logement et les droits de stockage des marchandises venant de l'extérieur afin que les hôteliers ne se profitent pas indûment des marchands étrangers. Ceux-ci devaient payer 6 sous par ballot et par nuit. Si les ballots se vendaient chez l'aubergiste, le marchand ne devait que 3 sous et s'ils s'associaient par moitié, il ne payait pas le logement. Les marchands se plaignaient toutefois des abus des aubergistes. En 1509, suite à une plainte de marchands anglais, bretons, galiciens et portugais accusant les hôteliers de leur acheter les marchandises à un trop bas prix, le conseil municipal de Bilbao interdit à ces derniers ce genre de pratique sous peine d'une amende de 2500 maravedis ${ }^{57}$ 
22 La plupart des ordonnances limitaient la pratique du commerce de détail par les étrangers, sauf permission expresse du conseil urbain. La coutume du Santander (1187) établit que «celui qui n'était pas voisin de la ville ne pourrait pas vendre au détail la marchandise de tissus portée par la mer, excepté aux voisins; et si l'etranger vendait au détail à un étranger, il devrait payer 10 sous $^{58}$ ». De cette façon les marchands locaux étaient les seuls intermédiaires entre les étrangers et la clientèle locale. Les métiers les mieux organisés, tels les tailleurs et les pêcheurs, se sont efforcés de faire appliquer ces dispositions. En 1500, Pierre Santos, voisin de San Vicente de la Barquera, tailleur et procureur de la confrérie de Saint-Bartolomé, informa le conseil municipal que les étrangers vendaient du tissus au détail dans la ville. Les accusés se défendirent en alléguant qu'ils le vendaient aux aubergistes pour compenser leurs frais de séjour. Le conseil municipal ordonna que, en vertu des ordonnances de la ville, les étrangers puissent vendre les tissus de laine durant huit jours seulement à partir de leur arrivée ${ }^{59}$. Les ordonnances de Bilbao de 1477 défendaient l'acquisition du fer au détail. Il fallait en acheter au minimum 50 quintaux et les voisins ne pouvaient pas le revendre ensuite aux marchands étrangers ${ }^{60}$. En 1515, un conflit éclata entre les fabricants de tissus de Bilbao ( plumeros) et «les Bretons habitant Bilbao » qui vendaient des étoffes aux voisins. Bien qu'ils soient habitants de la ville, le conseil municipal défendit que «Bretons ni Français ou d'autres étrangers n'osent vendre au détail ${ }^{61}$ ».

Les gouvernements urbains ont limité l'activité des étrangers pour la vente des deux produits plus importants dans les villes portuaires: le poisson et le vin. En 1504, la confrérie de pêcheurs de Lequeitio se plaignait de ce que le poisson importé par les étrangers les obligeait à baisser leur prix de vente. La municipalité décida que le poisson étranger ne pourrait se vendre qu'en temps de disette. Les étrangers devaient informer préalablement le conseil municipal pour vendre le poisson et respecter les prix locaux ${ }^{62}$. Les voisins ne pouvaient pas servir d'intermédiaires. En 1509, le conseil municipal de Bilbao établit que les marchands étrangers qui voulaient vendre du poisson devaient en donner un tiers au conseil municipal qui le livrerait aux regratières de la ville ${ }^{63}$. En ce qui concerne le vin, les conseils municipaux ont limité les importations par les étrangers. San Vicente de la Barquera défendit l'entrée du «vin étranger, blanc ou rouge, [...] sans permission du conseil municipal ${ }^{64} »$.

24 Les ordonnances municipales réglaient aussi le comportement des étrangers pendant leur séjour dans la ville en vue de protéger les voisins. En 1435, le conseil municipal de Bilbao décida que si un étranger défiait un voisin, il devait être expulsé de la ville et obligé de porter son défi hors de la ville sous peine de n'être plus y être admis ${ }^{65}$. En 1477, Bilbao défendait aux étrangers d'entrer dans la ville avec des armes ${ }^{66}$.

Malgré tous les contrôles que les villes imposaient aux étrangers, les gouvernements urbains savaient que le commerce avec eux était vital pour l'activité économique et pour la survie même de leur ville. En marge de la politique internationale des monarques, les villes portuaires ne pouvaient pas se passer des relations avec les villes atlantiques, en particulier Bayonne et Bordeaux. Dès le début du XIII ${ }^{\mathrm{e}}$ siècle, les ports de Gascogne ont passé des accords, soit individuellement soit en groupe. En 1306 et 1309, Castro Urdiales, Laredo et Santander signèrent un traité avec Bayonne, suivies par les villes du Guipúzcoa en 1309,1311 et $1328^{67}$. En 1351 et 1353, deux accords furent établis entre l'Angleterre et «Toutes Marismes \& Costeres de Meer, Portz, Citees \& Villes de la Seignurie du Roi de Castelle \& du Counté de Viscaye ${ }^{68} »$. En 1388, Saint-Sébastien accorda une lettre de protection générale à tous les Bretons arrivant avec des marchandises ${ }^{69}$. En 1404 et 1407, 
tous les ports « au bord de la mer de l'Espagne » et les ports de Gascogne signèrent des accords pour protéger les marchands et les navigateurs étrangers en temps de guerre comme de paix. En cas de guerre entre les rois de l'Angleterre et de la Castille, les marchands et les maitres de navires disposaient de quarante jours pour partir libres et en paix. Les villes portuaires castillanes développèrent leur propre stratégie politique et commerciale en direction des ports anglais et français les plus actifs et cherchèrent à se passer des autres pays qui pouvaient profiter du contexte politique pour s'introduire sur ces marchés.

L'un des moyens pour favoriser l'intégration des étrangers dans la société castillane était leur naturalisation. Selon le préambule du Titre IV de l'Espéculo d'Alphonse x (vers 1258) les étrangers pouvaient acquérir la nationalité castillane par la propriété, l'adoption, l'hérédité de sang ou après avoir vécu plus de deux ans sur le territoire. Une autre voie était le mariage avec une Castillane, bien que ce ne soit pas une condition indispensable. En général, les étrangers eurent une position ambiguë par rapport à la naturalisation. Celle-ci les intéressait parce qu'elle leur permettait de s'intégrer dans la vie politique et sociale, mais elle les empêchait de jouir des avantages économiques accordés par le roi aux étrangers et les obligeait à contribuer à la fiscalité municipale. Cornelis Deque, marchand néerlandais établi à Valladolid et possédant des intérêts économiques à Bilbao et Laredo, eut deux enfants, une fille avec Sancha de Bilbao de Bayçaval et un fils avec Catalina de Ronsa, de Bruges. Ce dernier se maria avec Francisca Quibetet, d'origine flamande ou bourguignonne. Dans le conflit l'opposant à Ferdinand del Hoyo, marchand de Laredo, Cornelis Deque fut accusé de ne pas vouloir se marier avec une Castillane pour ne pas devenir voisin : «Il a publiquement dit que bien qu'ils lui donnassent une femme avec une grande présence dans ces royaumes, qu'il ne la prendrait pas pour épouse pour ne pas devenir voisin ${ }^{70}$. » En général, les étrangers en Castille préférèrent se marier avec quelqu'un de la même nationalité pour une double raison, identitaire et économique, car il était plus rentable de rester étranger que de devenir Castillan, cela d'autant plus que le roi garantissait la protection des étrangers en Castille.

\section{NOTES DE FIN}

1. FINOT, Jules, Étude historique sur les relations commerciales entre la Flandre et l'Espagne du Moyen Âge. París, 1899 ; CAUNEDO DEL POTRO, Betsabe, La actividad de los mercaderes ingleses en Castilla (1475-1492), Madrid, 1984.

2. BELLO LEÓN, José Manuel, Extranjeros en Castilla (1474-1501). Notas y documentos para el estudio de su presencia en el reino a fines del siglo XV, La Laguna, 1994 ; FAGEL, Raymond, De Hispano-Vlaamse Wereld. De contacten tussen Spanjaarden en Nederlanders, 1496-1555, Bruxelles, 1996 ; ASENJO GONZÁLEZ, Maríaet IGUAL, David, « Mercaderes extranjeros en Valladolid. Una ciudad entre dos mares 1475-1500 », La Península Ibérica entre el 
Mediterráneo y el Atlántico, siglos XIII-XV, V Jornadas Hispano-portuguesas de Historia Medieval, Cadiz, 2006, p. 54-72 ; VALDEON BARUQUE, Julio, « Las colonias extranjeros en Castilla al sur del Tajo (los italianos en Andalucía en la Baja Edad Media) ", Anuario de Estudios Medievales, t. 10,1980 , p. $487-503$.

3. SOLÓRZANO TELECHEA, Jesús Ángel, Santander en la Edad Media : patrimonio, parentesco y poder, Santander, 2002 ; TENA GARCÍA, Soledad, La Sociedad urbana en la Guipúzcoa costera medieval : San Sebastián, Rentería y Fuenterrabía (1200-1500), Saint-Sébastien, 1997, p. 382-388 ; HELFFERICH, M. M. A. et CLERMONT, G., Fueros francos. Les communes françaises en Espagne et en Portugal pendant le Moyen Âge, Paris, 1860.

4. FAGEL, Raymond, «Corniles Deque, un mercader flamenco en la Castilla del siglo XV. Un debate sobre el concepto de «vecindad» $\mathrm{y}$ «naturaleza» entre mercaderes », Castilla y Europa. Comercio y Mercaderes en los siglos XIV, XV y XVI, Burgos, 1995, p. 241-264.

5. CHILDS, Wendy, «Commercial relations between the Basque provinces and England in the Latter Middles Ages. Ca. 1200-ca. 1500 ", Itsas, Memoria. Revista de Estudios marítimos del País Vasco, n 4, 2003, p. 62.

6. Voisin : statut juridique d'un citadin équivalent à celui de bourgeois.

7. Fuentes medievales del Archivo municipal de Mutriku (1237-1520), Saint-Sébastien, 2007, p. 322.

8. Archivo Real Chancillería de Valladolid, Reales Ejecutorias, caja 368/28.

9. Archivo de la Real Chancillería de Valladolid, Juzgado Mayor de Vizcaya, Pleitos civiles (olvidados), cajas 4421/5, 4232/7 y 4342/5. Archivo de la Real Chancillería de Valladolid, Juzgado Mayor de Vizcaya, Pleitos civiles (depositados), c. 4821/2. CAUNEDO DEL POTRO, Betsabe, Mercaderes castellanos en el Golfo de Vizcaya (1475-1492), Madrid, 1983, p. 95.

10. CASADO ALONSO, Hilario, « El comercio internacional castellano en tiempos de Isabel La Católica ", Isabel La Católica y su época. Actas del congreso internacional, vol. I, Valladolid, 2007, p. 651-682. STABEL, Peter, BLONDE, Bruno et GREVE, Anke (éd.), International trade in the Low countries (14 ${ }^{\text {th }}-16^{\text {th }}$ centuries). Merchants, Organisation, Infrastructure, Leuven, 2000.

11. Archivo General de Simancas, Registro General del Sello, vol. XI, fo 288. Désormais : AGS, RGS.

12. Colección documental del Archivo Histórico de Bilbao (1514-1520), p. 1516.

13. AGS, RGS, vol. IX, 1504, 04, 27.

14. AGS. Cámara de Castilla. Memoriales. Leg. 150, doc. 252.

15. CLASSEN, Albrecht (éd.), Meeting the Foreign in the Middle Ages, New York, 2002.

16. CANNY, Nicholas (éd.), Europeans on the move : studies on European Migration, 1500-1850, Oxford, 1994. PINOL, Jean-Luc (dir.), Les Immigrés et la ville. Insertion, intégration, discrimination, $\mathrm{XII}^{\mathrm{e}}-\mathrm{XX}^{\mathrm{e}}$ siècles, Paris, 1999.

17. SOLÓRZANO TELECHEA, Jesús Angel, « La fundación y promoción de las villas nuevas en litoral Atlántico del Norte peninsular durante el reinado de Alfonso X », El mundo urbano en la Castilla del siglo XIII, vol. II. Séville, p. 315-328.

18. Las Partidas, II, titre $X$, loi 3.

19. Memorial Histórico Español : colección de documentos, opúsculos y antigüedades que publica la Real Academia de la Historia, Madrid, 1951, t. II, p. 29.

20. RUIz, Teófilo F., « Trading with 'Other': economic exchanges between Muslims, Jews and Christians in the Late Medieval Castile ", collins, R. et Goodman, A. (éd.), Medieval Spain. Culture, conflict and coexistence. Studies in Honour of Angus Mackay, New York, 2002, p. 63-78.

21. AGS, RGS, vol. III, fol. 93. 
22. Ordenanzas reales de Castilla. Nuevamente corregidas de muchos vicios y faltas. Compiladas por Alfonso Díaz de Montalvo e impresas en Salamanca el 20 de octubre de 1541. 1779, p. 101. 23. Cortes de los antiguos reinos de León y de Castilla, t. III, Madrid, 1866, p. 18-19.

24. Libro de las Bulas y Pragmáticas de los Reyes Católicos, vol. II, Madrid, 1973, p. 316-318. Les quatre Villes de la Côte de la Mer étaient Castro Urdiales, Laredo, Santander et San Vicente de la Barquera.

25. Archivo General de Simancas. Registro General del Sello. Vizcaya (1484), Saint-Sébastien, 2003, p. 165.

26. AGS, Cámara de Castilla. Pueblos. Legajo 10, documento 609.

27. Real Academia de la Historia. Colección Vargas Ponce, t. XXIII, f 83-84.

28. Libro de las Bulas..., op. cit., p. 228.

29. Ibidem, p. 298-301.

30. Archivo de la Real Chancillería de Valladolid, Reales Ejecutorias, c. 216/3.

31. Colección documental del Archivo Histórico de Bilbao (1514-1520), vol. 4, Saint-Sébastien, 1999, p. 1544-1546.

32. Archivo General de Simancas. Registro General del Sello. Vizcaya (1478-1479), Saint-Sébastien, 2002, p. 66.

33. AGS, RGS, vol. I, fo $366 ; 1476,05,11$.

34. SUÁREZ FERNÁNDEZ, Luis, Política internacional..., op. cit., p. 375.

35. SUÁREZ FERNÁNDEZ, Luis, Política internacional..., op. cit., p. 444.

36. Colección documental del Archivo Histórico de Bilbao (1501-1514), Saint-Sébastien, 2000,

p. 1021.

37. SOLÓRZANO TELECHEA, Jesús Ángel, Colección documental de Santander en el Archivo General de Simancas (1326-1498), Santander, 1999, doc. 62.

38. Archivo General de Simancas. Registro General del Sello. Vizcaya (1480-1482), Saint-Sébastien, 2003, p. 163-170.

39. AGS, RGS, vol. V, 1489, 05, 24.

40. CAUNEDO DEl POTRO, Betsabé, Mercaderes castellanos..., op. cit., p. 225.

41. Archivo General de Simancas. Registro General del Sello. Vizcaya (1483), SaintSébastien, 2002, p. 79.

42. ORELLA UNZÚE, José Luis, Libro viejo de Guipúzcoa del bachiller Juan Martínez de Zaldivia, Saint-Sébastien, 1991, p. 187.

43. AGS, RGS, vol. XI, fo 133.

44. Cortes..., op. cit., vol. III, p. 15-16.

45. Ibidem, vol. III, p. 53 et 92.

46. SOlóRZANO TELECHEA, Jesús Angel, « Linaje, comunidad y poder: desarrollo y consolidación de identidades urbanas contrapuestas en la Castilla bajomedieval », Familia y sociedad en la Edad Media (siglos XII-XVI), Zaragoza, 2007, p. 71-93.

47. Archivo de la Real Chancillería de Valladolid, Reales Ejecutorias, c. 286/32, $\mathrm{f}^{\circ} 3 \mathrm{v}^{\circ}-4 \mathrm{v}^{\circ}$. 48. BARÓ PAZOS, Juan et GALVÁN RIVERO, Carmen, Libro de ordenanzas de la villa de Castro Urdiales (1519-1572), Santander, 2006, p. 192 et 193.

49. MOgRo, Juan José, Laredo-Santoña. Sus pleytos. Santander, 2001, p. 43.

50. BARÓ PAZOS, Juan et GALVÁN RIVERO, Carmen, Libro Castro Urdiales..., op. cit., p. 115.

51. Archivo Municipal de Deba (1181-1520), t. I., Saint-Sébastien, 2006, p. 107.

52. GUIARD Y LARRAURI, Teófilo, Historia de la noble villa de Bilbao (1300-1600), t. I, Bilbao, 1971, p. 438 et 470 .

53. BARÓ PAZOS, Juan et GALVÁN RIVERO, Libro Castro Urdiales..., op. cit, p. 124. 
54. Ordenanzas municipales del Bilbao (1477-1520)..., op. cit., p. 220.

55. GUAL CAMARENA, Miguel, «El hospedaje hispano-medieval. Aportaciones para su estudio », Anuario de Historia del Derecho Español, t. XXXII, 1962, p. 527-542.

56. Archivo General de Simanca. Registro general del sello. Vizcaya (1480-1482), Saint-Sébastien, 2002, p. 163-165.

57. Libro de acuerdos de Bilbao (1509-1515), Saint-Sébastien, 1999, p. 49-50.

58. SOLÓRZANO TELECHEA, Jesús Angel, «El fenómeno urbano medieval en Cantabria », El fenómeno urbano medieval entre el Cantábrico y el Duero, Santander, 2002, p. 293.

59. AGS. Cámara de Castilla. Pueblos. Leg. 17, doc. 338.

60. Ordenanzas municipales del Bilbao (1477-1520)..., op. cit., p. 21, 23, 26, 48-49.

61. Libro de acuerdos de Bilbao (1509-1515)..., op. cit., p. 308.

62. Colección documental del archivo de la cofradía de pescadores de la villa de Lequeitio. San Sebastián, 1991.

63. Libro de acuerdos y decretos municipales de la villa de Bilbao (1509 y 1515), Saint-Sébastien, 1995, p. 156.

64. Archivo de la Real Chancillería de Valladolid, Pleitos civiles, Alonso Rodríguez, c. $786 / 1, \mathrm{f}^{\circ} 1 \mathrm{v}^{\circ}$.

65. TEÓFILO, GuiardLarrauri, Historia de la noble villa de Bilbao..., op. cit.,p. 109.

66. Ordenanzas municipales del Bilbao (1477-1520), Saint-Sébastien, 1995, p. 26.

67. SolóRzAnO TelecheA, Jesús Angel, Patrimonio documental de Santander..., op. cit., documents 18-22.

68. RYMER, T., Foedera, convenciones, literae et cuiuscunque generis acta publica inter reges Angliae et alios quosvis imperatores, reges, pontifices, principes vel communitates, vol. VI, Londres, 1727, p. 29.

69. CAMINO Y ORELLA, Joaquín, Historia civil-diplomática-eclesiástica antigua y moderna de la ciudad de San Sebastián, Saint-Sébastien, 1963, p. 280.

70. AGS, Cámara Castilla, Personas, 13. Hoyo (Fernando del) y otros.

\section{RÉSUMÉS}

Cette étude aborde en premier lieu la présence des étrangers dans les villes portuaires du Nord de la Couronne de la Castille depuis la deuxième moitié $\mathrm{du}$ XII ${ }^{\mathrm{e}}$ siècle jusqu'au début $\mathrm{du} \mathrm{XVI}^{\mathrm{e}}$ siècle. Elle s'intéresse ensuite à la législation royale destinée à protéger les étrangers et à régler leurs activités économiques tandis que les autorités des villes portuaires, au moyen d'ordonnances municipales, tentaient de contrôler la présence et les activités de ces derniers.

Firstly, this essay researches the presence of the foreigners in the seaports on the Northern coast of Spain from the second half of the $12^{\text {th }}$ century to the beginning of the 16th century. Secondly, we will expound the royal legislation in the Kingdom of Castile in relation to the protection of foreigners and the regulation of their economic activities. Finally, we will explain the role played by the town councils to control the foreign presence and to regulate the economic activities by means of municipal ordinances.

\section{INDEX}

Thèmes : Castille

Index chronologique : Moyen Âge 


\section{AUTEURS}

\section{JESÚS ÁNGEL SOLÓRZANO TELECHEA}

profesor titular de historia medieval Universidad de Cantabria

BEATRIZ ARÍZAGA BOLUMBURU

catedrática de historia medieval Universidad de Cantabria 